\title{
3D Mold Fabrication Techniques Using an Inorganic Resist
}

\author{
J. Taniguchi, Y. Taguchi", Y. Sugiyama ${ }^{*}$, S. Ono ${ }^{*}$, and I. Miyamoto \\ Department of Applied Electronics, Tokyo University of Science, 2641 Yamazaki, Noda, Chiba 278-8510 Japan \\ "ELIONIX, 3-7-5 Motoyokoyamachou, Hachioji, Tokyo 192-0063 Japan
}

\begin{abstract}
We developed nanometer-order fabrication for 3-dimensional (3D) imprint molds, using acceleration-voltage-modulation electron beam (EB) direct writing. The inorganic EB resist was spin-on-glass (SOG), whose depth was controlled by changing the EB acceleration voltage. After EB exposure, the sample was developed using buffered hydrofluoric acid. The fabricated pattern depths on SOG were well gradated, and the depth resolution was $20 \mathrm{~nm}$ per $100 \mathrm{~V}$. The width resolution was $40 \mathrm{~nm}$ on SOG using an electron beam with a diameter of a few nanometers. Patterns were transferred by pressing the fabricated $3 \mathrm{D}$ SOG mold to a photo-curable resin under a pressure of $0.5 \mathrm{MPa}$ and curing it with a $1 \mathrm{~J} / \mathrm{cm}^{2}$ ultraviolet dose. Replicated patterns showed faithful, defect-free multigradation. Using SOG as the material for an ion beam etching mask, 3D molds were fabricated from diamond, engineering plastic, and quartz.
\end{abstract}

Key words: nanoimprint lithography, photo-curable resin, three-dimensional imprint, inorganic resist, spin-on glass, electron beam lithography, buffered hydrofluoric acid

\section{Introduction}

Nanoimprint lithography (NIL) is a major breakthrough in nanopatterning, because it produces nanometer features over a large area with high throughput and at low $\operatorname{cost}^{1,2)}$. NIL studies have included diamond mold imprint lithography ${ }^{3), 4)}$, sapphire mold imprint lithography ${ }^{5)}$, and imprint lithography using ultraviolet (UV) curable resin for high throughput ${ }^{6), 7)}$, all of which use 2-dimensional pattern transfer. In view of the need for nano-order 3-dimensional (3D) fabrication in various applications such as photonic crystals ${ }^{8)}$, binary optical elements, and 3D storage media, we developed 3D electron beam (EB) lithography using an inorganic resist, as a result of which we attained nanometer resolution and nanometer-order depth gradation. In the sections that follow, we give details 3D of our mold fabrication techniques and the patterns we transferred to photo-curable resin.

\section{Experimental Apparatus and Procedures}

We used Accuglass 512B, an SOG material which mainly consists of siloxane with $14 \%$ organic content, whose interlayer dielectrics were developed by Honeywell Co. We found that Accuglass 512B behaves as a positive inorganic EB resist when used with buffered hydrofluoric acid (BHF) in development. The BHF developing solution was mixed with $50 \% \mathrm{HF} 25 \mathrm{~cm}^{3} /$ liter and $40 \% \mathrm{NH}_{4} \mathrm{~F} 30$ $\mathrm{cm}^{3} /$ liter. The calculated concentration of [HF] was 0.43 $\mathrm{mol} / \mathrm{liter}$ and that of $\left[\mathrm{HF}_{2}{ }^{-}\right]$was $0.29 \mathrm{~mol} / /$ liter. The SOG resist depth was controlled by changing the EB acceleration voltage, which enabled us to vary the projected electron range, using a low acceleration voltage to make shallow pits and a high acceleration voltage to make deep pits. We were thus able to fabricate different depth-gradation 3D molds by means of EB exposure. The EB exposure system consisted of a conventional scanning electron microscope (SEM) with a PC-based EB drawing system. To obtain sufficient EB current at a low acceleration voltage of $1-5 \mathrm{kV}$, we shortened the distance between the anode and EB filament by $5 \mathrm{~mm}$. Using this technique, a 1.4 times larger electron beam current was obtained. When the EB acceleration voltage was changed from $1 \mathrm{kV}$ to $5 \mathrm{kV}$, the exposure current was a few $\mathrm{nA}$ and the beam diameter was almost constant. Fine pattern EB writing was performed by using an ELS-7500 (Elionix Co.), which can provide a beam with a diameter of a few nanometers. PAK01 (blended by Toyo Gosei Co., Ltd.), used as the photo-curable resin, is an acryl resin composed mainly of tripropylene-glycol-diacrylate monomer with dimethoxy-phenyl-acetophenon as a photoinitiator. In 3D mold fabrication (Figure 1), (1) SOG was spin-coated on a Si substrate and cured at $300^{\circ} \mathrm{C}$ for 1 hour, after which the SOG was $500 \mathrm{~nm}$ thick. (2) The sample was drawn by an EB exposure system with a $500 \mu \mathrm{C} / \mathrm{cm}^{2}$ dose at different acceleration voltages. In the case of SOG resist, the developed depth was not changed for doses ranging from 200 to $1000 \mu \mathrm{C} / \mathrm{cm}^{2}$, and therefore we used a constant value. The sensitivity of SOG is low, and thus it takes a long time to expose. This means that the productivity of molds using SOG resist is low. However, fabrication of high-sensitivity SOG resists, such as chemically amplified resists, is possible if we design specially for SOG. (3) After EB exposure, the sample was developed by applying BHF solution for $60 \mathrm{~s}$ to remove EB-exposed SOG; this enabled us to fabricate SOG molds with different depth gradations (3D mold).

Figure 2 shows the imprint process using a 3D mold. (1) The fabricated 3D mold was treated with Aquaphobe CF (Gelest Co.), which is a silane coupling oligomar considered by the University of Texas as a good release coating material because of its low surface energy ${ }^{9}$. Then, photo-curable resin was dropped onto the $3 \mathrm{D}$ mold. (2) The 3D mold was pressed onto the slide glass with a pressure of $0.5 \mathrm{MPa}$. (3) The photo-curable resin was exposed to a $1 \mathrm{~J} / \mathrm{cm}^{2}$ dose of UV light through the slide 
glass and cured. (4) The 3D mold was released from slide glass, and a 3D replica pattern was obtained. To measure the precise depth dependence of the acceleration voltage change, we used a step profilometer ( $\alpha$-step). To enable the profilometer stylus to reach the bottom of the patterns, we set the drawing pattern width to $10 \mu \mathrm{m}$.

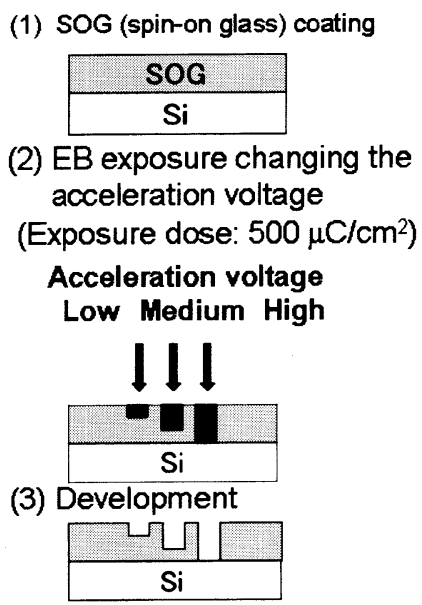

Fig. 1 Fabrication of 3D SOG mold.
(1) Drop photo-curable resin

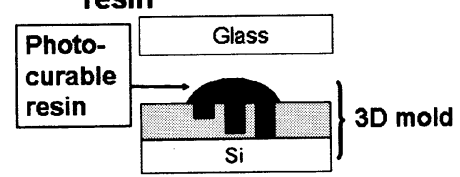

(2) Press mold

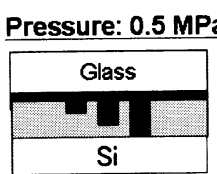

(3) UV exposure

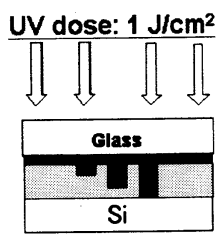

(4) Release mold

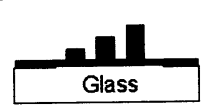

Fig. 2 Imprint process using a 3D mold and photo-curable resin.

We also fabricated 3D molds made of diamond, engineering plastic, and quartz, using SOG as a mask in ion beam etching (IBE). An electron cyclotron resonance (ECR) ion beam source was used in IBE. IBE was performed at a $6 \times 10^{-4} \mathrm{~Pa}$ base pressure and $100 \mathrm{~W}$ microwave power.

\section{Experimental Results and Discussion}

\subsection{D mold fabrication and pattern transfer}

Figure 3 shows a 3D SOG mold fabricated at acceleration voltages from $1 \mathrm{kV}$ to $5 \mathrm{kV}$, and an enlarged view of the pattern (Fig. 3 (b)). Patterned depths gradated well, with a depth differential between $1.7 \mathrm{kV}$ and $1.8 \mathrm{kV}$ patterns of $20 \mathrm{~nm}$, indicating a depth resolution of $20 \mathrm{~nm}$ per $100 \mathrm{~V}$. Figure 4 shows the patterns transferred by using a 3D SOG mold (a) and an enlarged view of the patterns in the elliptical area (b). Multigradation patterns are faithfully transferred with a height resolution of 20 $\mathrm{nm}$. This indicates that imprint technology is applicable to
3D fabrication, enabling high throughput and mass production of $3 \mathrm{D}$ products.

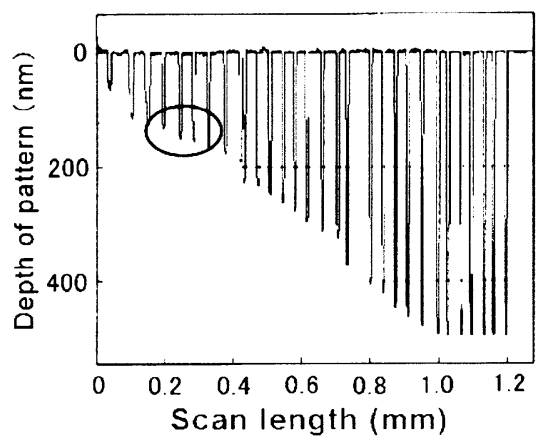

(a)

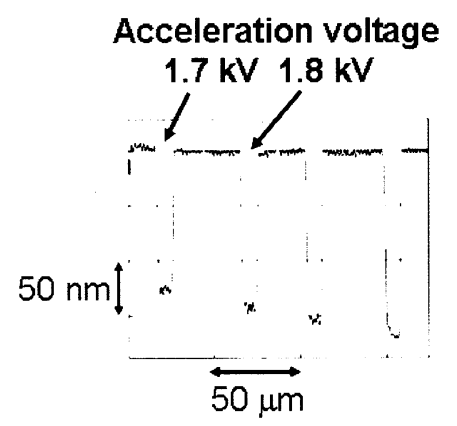

(b)

Fig. 3 Fabricated 3D SOG mold. (a) Whole fabricated patterns measured by the step profilometer. (b) Enlargement of the patterns shown in the elliptical area in (a).

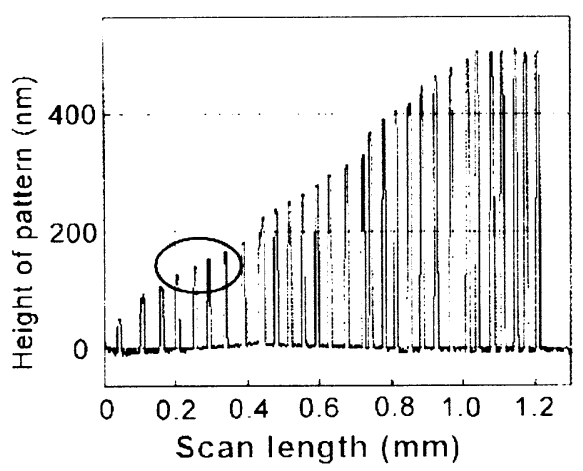

(a)

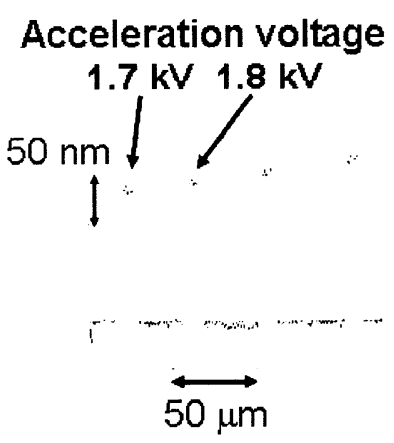

(b)

Fig. 4 Patterns transferred by using a 3D SOG mold: (a) Whole replicated patterns. (b) Enlargement of the patterns shown in the elliptical area in (a). 
Figure 5 compares the patterned mold depth and replicated pattern height, showing that they coincide well for each acceleration voltage. Both curves are saturated at $4 \mathrm{kV}$ because the patterned depth reached the initial SOG thickness of $500 \mathrm{~nm}$.

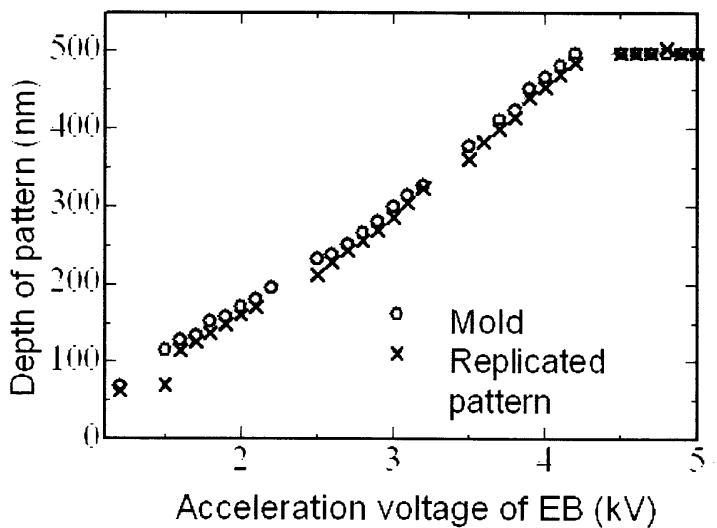

Fig. 5 Comparison of the patterned mold depth and replicated pattern height.

\subsection{Evaluation of the width resolution}

Figure 6 shows a 40-nm-wide pattern on SOG that was produced by using EB direct writing with EB diameters of a few nanometers. Furthermore, the line edge roughness of each line in Fig. 6 was small, and thus the SOG was also useful for EB cutting resist. Therefore, this technique has the potential for use in creating $3 \mathrm{D}$ products with sub-50-nm space resolution (both line width and depth gradation). Nanoimprint lithography reveals a potential for fabricating mold patterns for various polymers ${ }^{1)-7)}$ with nano-order replication and with high fidelity. Thus, accuracy and flexibility of the mold are the key points for high-value-added products. Now, nanoimprint techniques are widely used not only for lithography but also for molding processes. Using 3D patterned $\mathrm{SOG}$ for a dry-etching mask, molds of various shapes and materials are fabricated, and the flexibility of the process for fabrications molds is very important in various applications. In the following section, fabrication processes for $3 \mathrm{D}$ molds using various materials are described.

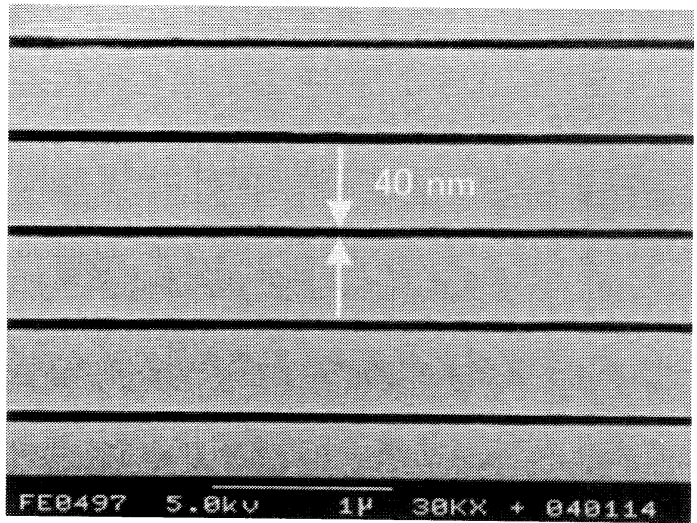

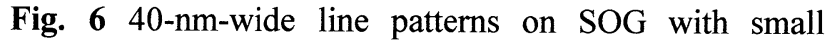
line-edge roughness.

\subsection{Fabrication of 3D diamond molds}

Diamond is a promising imprint mold candidate because of its high Knoop hardness, compressive strength, tensile strength, and thermal conductivity and its low thermal expansion coefficient. 3D diamond molds are consequently also attractive. In 3D diamond mold fabrication, (1) SOG was spin-coated on diamond and (2) after SOG curing, EB exposure was performed while changing the acceleration voltage. (3) The sample was then developed using BHF, and the 3D SOG pattern was obtained on diamond. (4) Using the SOG layer as a mask in oxygen ion beam etching, diamond was etched by oxygen ion beam bombardment until a 500-nm SOG layer was etched away. Figure 7 shows a fabricated 3D diamond mold with 5 depths of gradation, the deepest pattern at 850 $\mathrm{nm}$ corresponding to the 500-nm-deep SOG mask pattern; i.e., in this case, pattern depth was magnified 1.7 times. SOG is composed mainly of silicon dioxide, having good resistance to oxygen ion beam bombardment. Diamond, however, consists of carbon, and is consequently easily damaged by oxygen ion beam bombardment. Using SOG as the mask in oxygen ion beam etching of materials containing carbon, such as diamond, PMMA, SiC, and diamond-like carbon, enables pattern depths to be magnified: this offers an advantage over other dry etching processes, because 3D deep, high-aspect-ratio patterns are easily obtained without treatment after oxygen ion beam etching. In the following section, fabrication processes for $3 \mathrm{D}$ molds made of engineering plastic are described.

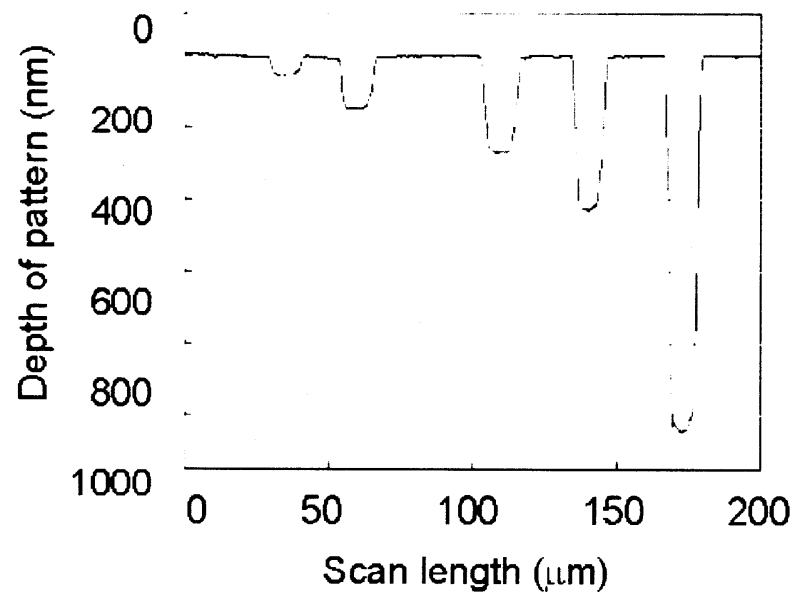

Fig. 7 Fabricated 3D diamond mold with 5 depth gradations.

\subsection{Fabrication of 3D engineering plastic molds}

PEI (poly-ether-imide) is a kind of engineering plastic, and this substrate was used for specimen. Engineering plastic has a high mechanical toughness and high thermal resistance in comparison with normal plastic, and consequently has the potential to be used for imprint molds. The fabrication procedure was similar to that in the case of diamonds. First, a $2 \mathrm{D}$ pattern was fabricated to measure the selectivity. Figure 8 shows SEM photographs of a fabricated PEI surface using a reactive ion beam of 
oxygen at $0.60 \mathrm{~mA} / \mathrm{cm}^{2}$ ion beam current density and $400 \mathrm{~V}$ acceleration voltage for $40 \mathrm{~min}$. As shown in the figure, deep grooves were formed in the PEI substrate. The thickness of the SOG mask was $535 \mathrm{~nm}$ and the obtained depth of PEI was $8.46 \mu \mathrm{m}$; thus, the selectivity is 15.8 . The figure also shows $1-\mu \mathrm{m}$-wide lines, whose aspect ratio was 8.46 . Therefore, this process can be used to fabricate a high-aspect- ratio mold.

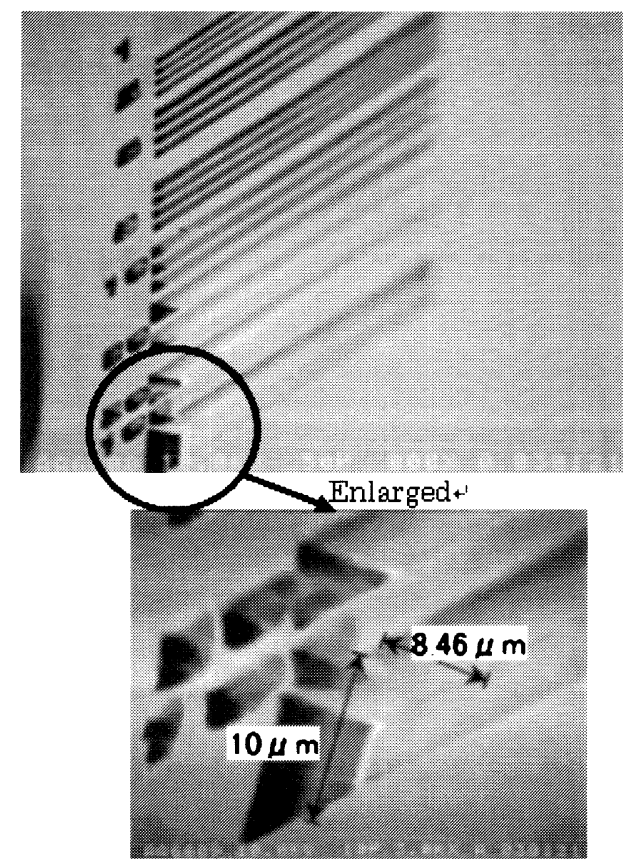

Fig. 8 SEM photographs of line patterns transferred onto the PEI substrate.

Usually, high-aspect-ratio patterns are fabricated by using the LIGA (lithographie, galvanoformung, and abformung) process. However, this process requires $\mathrm{X}$-ray radiation equipment and a precise X-ray mask, and these are expensive. Thus, the SOG mask process is cost-effective in comparison with the LIGA process. When a 3D SOG mask is used, a 3D high-aspect-ratio pattern is obtained, and consequently the flexibility of this mold fabrication process is very high. A 3D mold of PEI is shown in Fig. 9.

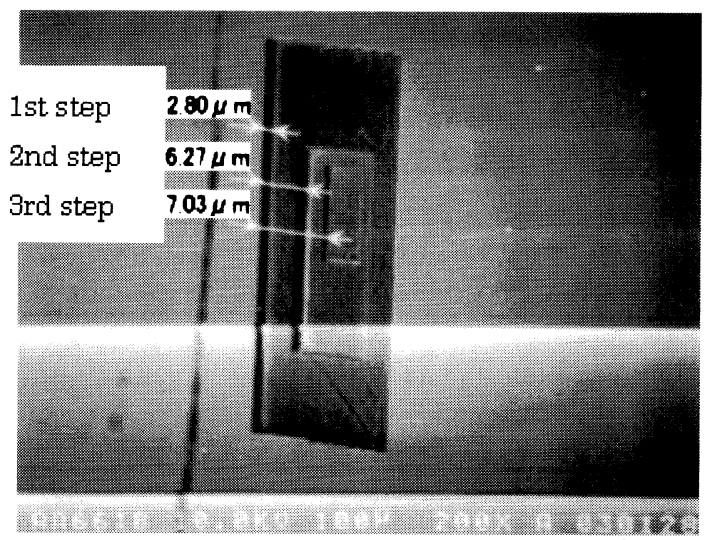

(a) SEM photograph of the pattern transferred onto the PEI substrate.

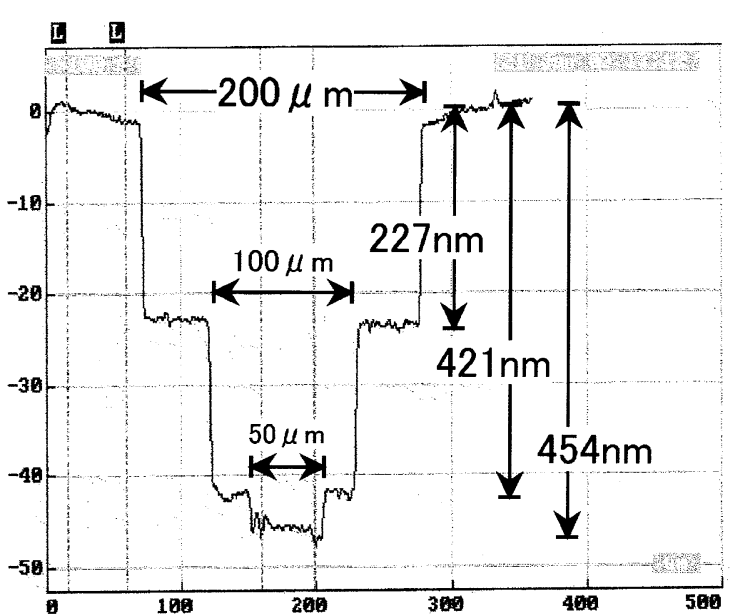

(b) Cross-section of the SOG mask

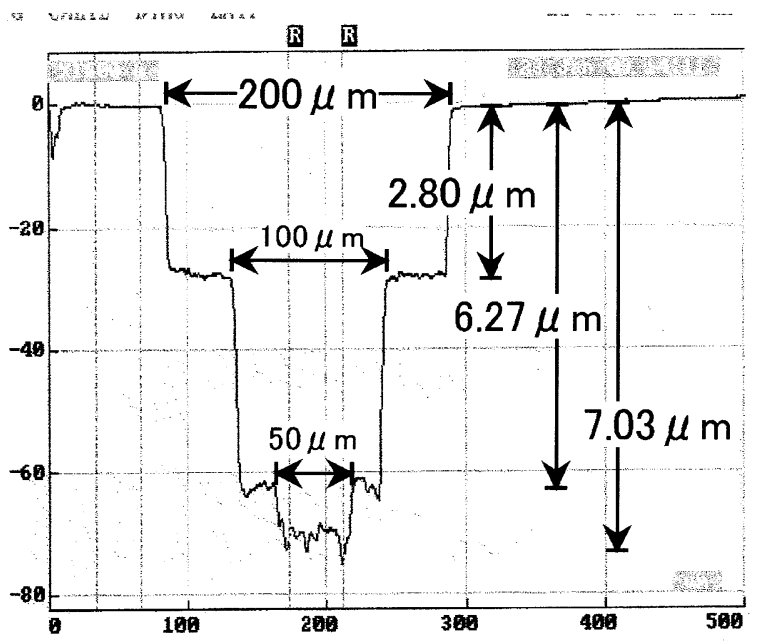

(c) Pattern transferred onto the PEI substrate

Fig. 9 SEM photograph of the pattern transferred onto the PEI substrate (a), cross-section of the SOG mask pattern (b), and the pattern transferred onto the PEI substrate (c).

As shown in these figures, three steps were formed in the PEI substrate. The depth is 15.5 times deeper than that of the SOG mask pattern, because of the high selectivity between the PEI substrate and the SOG film. These results show that this technique can be used for micro-electro-mechanical systems (MEMSs), micro-fluidic systems, and biochemical devices.

\subsection{Fabrication of 3D quartz molds}

Quartz molds are also important, because quartz is transparent to UV light and is used in UV imprint lithography ${ }^{5), 7)}$. Quartz is also an optimum material for micro-reactors because of its chemical resistance and high mechanical strength. However, it is difficult to fabricate 3D patterns on quartz efficiently. For example, injection molding of quartz is impossible, because quartz has a high melting point. Laser cutting of quartz is also difficult because of its transparency over a wide range of wavelengths. Nanoimprint molds and micro-reactors require a $3 \mathrm{D}$ structure for sophisticated purposes. For instance, a dam structure is utilized for immunoassay. 
Therefore, establishment of an efficient and precise 3D fabrication process for quartz is required. Using 3D patterned SOG for an ion beam etching mask, it is possible to fabricate $3 \mathrm{D}$ gradations on the surface of quartz. In order to examine SOG as an efficient mask for quartz, ion species and acceleration voltages were varied and etching rates were investigated. Figure 10 shows the relationship between the acceleration voltage and etched depth of SOG. Figure 11 shows the relationship between the acceleration voltage and etched depth of quartz. As shown in these figures, a $\mathrm{CF}_{4}$ ion beam at an acceleration voltage of $500 \mathrm{~V}$ has the highest selectivity (etched depth of quartz/ etched depth of SOG), whose value is 1.1. Thus, the etched depth of quartz was 1.1 larger than that of the SOG mask. Furthermore, in this condition, the etching rate was fast, so the transfer of patterns was completed immediately. Thus, this condition is optimum in terms of both mask efficiency and fabrication speed.

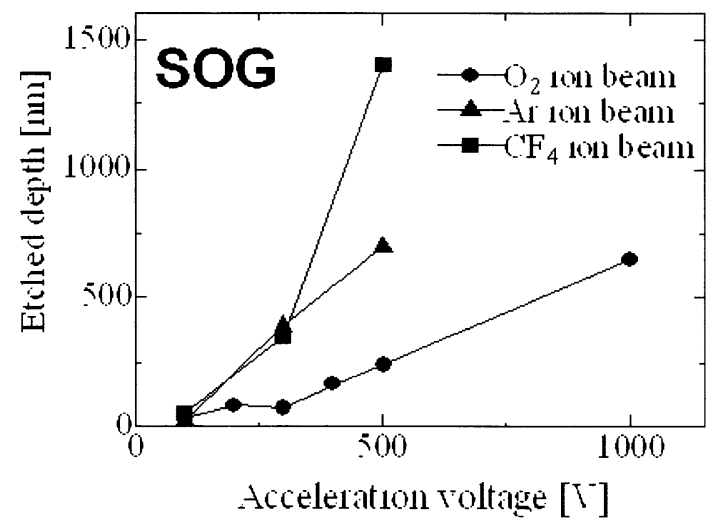

Fig. 10 Relationship between the acceleration voltage and the etched depth of SOG.

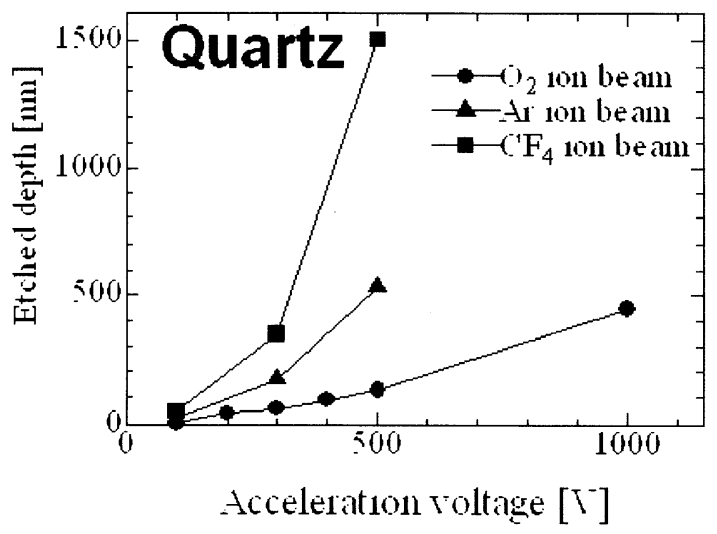

Fig. 11 Relationship between the acceleration voltage and the etched depth of quartz.

To fabricate 3D groove patterns onto a quartz surface, 3D SOG patterns were first fabricated on quartz, using accelerating-voltage-modulation EB exposure. Figure 12 shows a top view (a) and cross-sectional profile (b) of an SOG pattern. The depths of grooves were $276 \mathrm{~nm}$ at an EB accelerating voltage of $3 \mathrm{kV}, 407 \mathrm{~nm}$ at $4 \mathrm{kV}$, and 466 $\mathrm{nm}$ at $5 \mathrm{kV}$, respectively. The width of every groove was $10 \mu \mathrm{m}$. In the case of an acceleration voltage of $5 \mathrm{kV}$, the etched depth was saturated and was equal to the SOG thickness. Thus, in this case the SOG thickness was 466 nm.

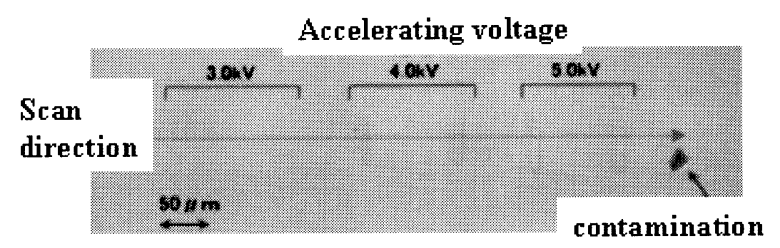

(a)

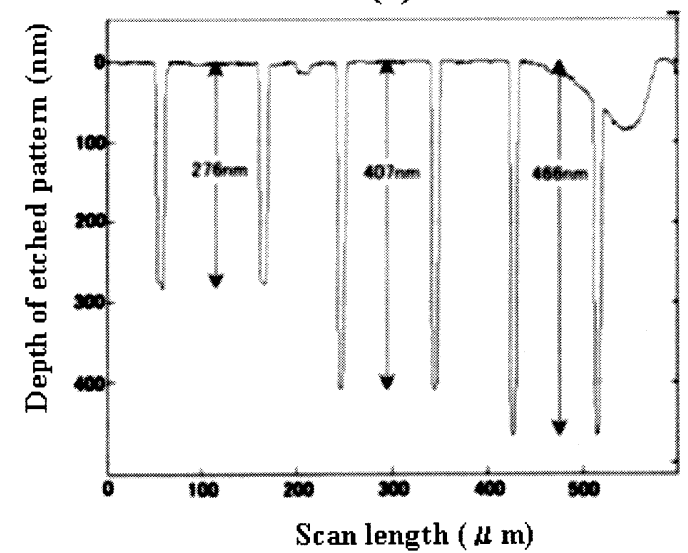

(b)

Fig. 12 Etched grooves of an SOG pattern on quartz substrate.

(a) Optical microscope image of the top view.

(b) Cross-sectional profile of etched grooves measured with a surface profilometer.

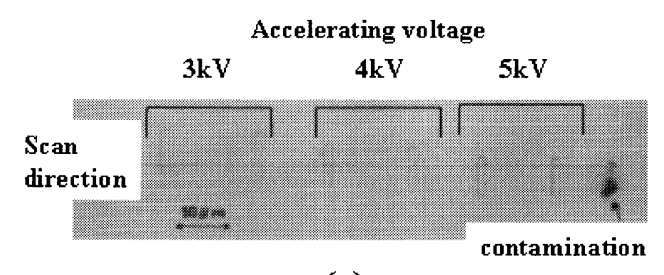

(a)

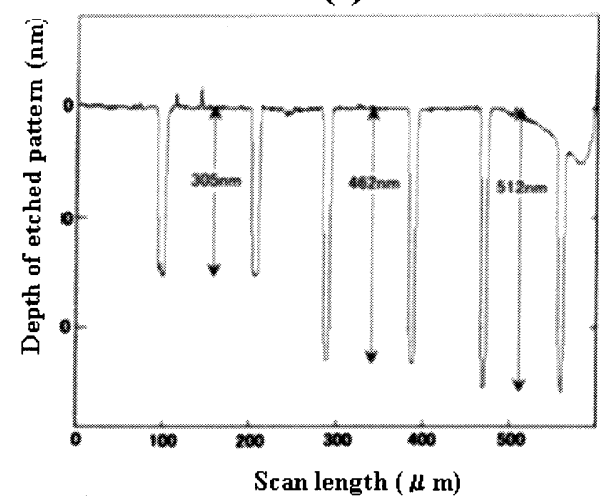

(b)

Fig. 13 Fabricated groove patterns on quartz surface.

(a) Optical microscope image of the top view.

(b) Cross-sectional profile of fabricated grooves measured with a surface profilometer. 
After the fabrication of a 3D mask of SOG, ion beam etching was carried out. The etching conditions were as follows: $\mathrm{CF}_{4}$ ion species, $500 \mathrm{~V}$ acceleration voltage, and 5 min etching time. According to Fig.10, 5 min etching yielded a $750 \mathrm{~nm}$ etching depth, which is larger than the initial SOG thickness $(466 \mathrm{~nm})$. To eliminate the cleaning process of quartz, the SOG mask was removed by ion beam bombardment. Therefore, a 5-min etching time was selected. Figure 13 shows the top view (a) and a cross-sectional profile (b) of the quartz surface. The depths of grooves were $305 \mathrm{~nm}$ at an EB accelerating voltage of $3 \mathrm{kV}, 462 \mathrm{~nm}$ at $4 \mathrm{kV}$ and $512 \mathrm{~nm}$ at $5 \mathrm{kV}$, respectively. Every groove has a selectivity value (etched depth of quartz / etched depth of SOG) of 1.1. Therefore, this method allows precise depth control and uniform pattern etching on a quartz surface.

\section{Conclusions}

We established a fabrication process for nanometer-order 3D imprint molds using a novel acceleration voltage modulation EB direct writing technique and inorganic EB resists. The fabricated pattern depths on SOG were gradated well, and this technique has a depth resolution of $20 \mathrm{~nm}$ per $100 \mathrm{~V}$. Furthermore, a width resolution of $40 \mathrm{~nm}$ on SOG was obtained. Imprint patterns were faithfully obtained with 20-nm height gradations, which suggests that mass-production of $3 \mathrm{D}$ nano-patterning is possible. Using SOG for ion beam etching mask, 3D molds were fabricated from diamond, engineering plastic, and quartz. These results indicate that it is possible to fabricate molds with flexible designs from various materials. Molds play an important role in nanoimprint technologies, and therefore these techniques raise the potential of nanoimprint technologies.

\section{Acknowledgments}

This work was supported by the project for Cooperation between Industry and Universities, organized by the Ministry of Education, Culture, Sports, Science and Technology.

It was also supported by research funds from the TEPCO Research Foundation and the Foundation of CASIO and Saneyoshi Shougakukai, and by the Ando Incentive Prize for the Study of Electronics. We thank Rasa Industries, Ltd., for preparation of materials.

\section{References}

1) S. Y. Chou, P. R. Krauss, and P. J. Renstrom: Appl. Phys. Lett. 67 (1995) 3114.

2) S. Y. Chou, P. R. Krauss, and P. J. Renstrom: J. Vac. Sci. \& Technol. B 14 (1996) 4129.

3) J. Taniguchi, Y. Tokano, I. Miyamoto, M. Komuro, H. Hiroshima, K. Kobayashi, T. Miyazaki and H. Ohyi: Jpn. J. Appl. Phys. 39 (2000) 7070.

4) J. Taniguchi, Y. Tokano, I. Miyamoto, M. Komuro, and H. Hiroshima, Nanotechnology 13 (October 2002) 592.

5) M. Komuro, Y. Tokano, J. Taniguchi, T. Kawasaki, I. Miyamoto, and H. Hiroshima: Jpn. J. Appl. Phys. 41 (2002) 4182.

6) M. Komuro, J. Taniguchi, S. Inoue, N. Kimura, Y. Tokano, H. Hiroshima, and S. Matsui: Jpn. J. Appl. Phys. 39 (2002) 7075.

7) J. Taniguchi, T. Kawasaki, Y. Tokano, Y. Kogo, I. Miyamoto, M. Komuro, H. Hiroshima, N. Sakai, and K. Tada: Jpn. J. Appl. Phys. 41 (2002) 4194.

8) E. Kuramochi, M. Notomi, T. Tamamura, T. Kawashima, S. Kawakami, J. Takahashi, and C. Takahashi: J. Vac. Sci. \& Technol. B 18 (2000) 3510.

9) T. Bailey, B. J. Choi, M. Colburn, M. Meissl, S. Shaya, J. G. Ekerdt, S. V. Sreenivasan, and C. G. Willson: J. Vac. Sci. \& Technol. B 18 (2000) 3572.

Received May 16, 2004; Accepted June 16, 2004. 